\title{
Automated generation of genome-scale metabolic draft reconstructions based on KEGG
}

\author{
Emil Karlsen ${ }^{1 \dagger}$, Christian Schulz ${ }^{1 \dagger}$ and Eivind Almaas ${ }^{1,2^{*}}$ (D)
}

\begin{abstract}
Background: Constraint-based modeling is a widely used and powerful methodology to assess the metabolic phenotypes and capabilities of an organism. The starting point and cornerstone of all such modeling is a genome-scale metabolic network reconstruction. The creation, further development, and application of such networks is a growing field of research thanks to a plethora of readily accessible computational tools. While the majority of studies are focused on single-species analyses, typically of a microbe, the computational study of communities of organisms is gaining attention. Similarly, reconstructions that are unified for a multi-cellular organism have gained in popularity. Consequently, the rapid generation of genome-scale metabolic reconstructed networks is crucial. While multiple web-based or stand-alone tools are available for automated network reconstruction, there is, however, currently no publicly available tool that allows the swift assembly of draft reconstructions of community metabolic networks and consolidated metabolic networks for a specified list of organisms.
\end{abstract}

Results: Here, we present AutoKEGGRec, an automated tool that creates first draft metabolic network reconstructions of single organisms, community reconstructions based on a list of organisms, and finally a consolidated reconstruction for a list of organisms or strains. AutoKEGGRec is developed in Matlab and works seamlessly with the COBRA Toolbox v3, and it is based on only using the KEGG database as external input. The generated first draft reconstructions are stored in SBML files and consist of all reactions for a KEGG organism ID and corresponding linked genes. This provides a comprehensive starting point for further refinement and curation using the host of COBRA toolbox functions or other preferred tools. Through the data structures created, the tool also facilitates a comparative analysis of metabolic content in any given number of organisms present in the KEGG database.

Conclusion: AutoKEGGRec provides a first step in a metabolic network reconstruction process, filling a gap for tools creating community and consolidated metabolic networks. Based only on KEGG data as external input, the generated reconstructions consist of data with a directly traceable foundation and pedigree. With AutoKEGGRec, this kind of modeling is made accessible to a wider part of the genome-scale metabolic analysis community.

Keywords: AutoKEGGRec, Genome-scale metabolic model, Constraint-based analysis, Pipeline, Draft model, Metabolic network reconstruction, Multiple organisms, Community model, Consolidated model, COBRA

*Correspondence: eivind.almaas@ntnu.no

†Emil Karlsen and Christian Schulz contributed equally to this work.

${ }^{1}$ Department of Biotechnology and Food Science, NTNU - Norwegian

University of Science and Technology, Høgskoleringen 1, 7491 Trondheim,

Norway

${ }^{2}$ K.G. Jebsen Center for Genetic Epidemiology, Department of Public Health

and General Practice, NTNU - Norwegian University of Science and

Technology, Trondheim, Norway

(c) The Author(s). 2018 Open Access This article is distributed under the terms of the Creative Commons Attribution 4.0 International License (http://creativecommons.org/licenses/by/4.0/), which permits unrestricted use, distribution, and reproduction in any medium, provided you give appropriate credit to the original author(s) and the source, provide a link to the Creative Commons license, and indicate if changes were made. The Creative Commons Public Domain Dedication waiver 


\section{Background}

Genome-scale metabolic modeling has gained a sharp increase in popularity in recent years. Currently, there exist more than 160 well curated metabolic network reconstructions [1] spanning more than 100 species, such as the Bacteria Bacillus, Clostridium, Escherichia, Pseudomonas, and Salmonella, the Eukaryota Homo sapiens, and Saccharomyces, and the Archaea Methanosarcina, to name just a few. While many of these reconstructions were created years ago, they are still a helpful and reliable source when constructing a new genome-scale metabolic model (GEM) for another species, or to extend the knowledge of an existing reconstruction. However, the metabolic networks are often reconstructed using quite different methodologies and approaches with a wide variety of annotation conventions. Consequently, the comparison of genome-scale reconstructions is not straightforward when the interest is in identifying how much a set of reconstructed networks actually differ in biological content. This challenge needs to be addressed by the modeling community in order to avoid unnecessary repetition of arduous reconstruction work $[2,3]$.

For manipulating and curating these model metabolic networks, different tools exist. Most notable is the COBRA Toolbox [4], an open-source community-driven software suite available in Matlab. This toolbox contains a large number of built-in functions for the analysis and refinement of constraint-based metabolic models. Having found numerous and potent applications within modeling and analysis in fields such as biology and medicine, it remains a popular and well-supported platform for such work $[4,5]$. However, it currently contains no tools for the rapid assembly of large-scale first-draft models.

The development of tools to aid and assist in the reconstruction of GEMs has recently been an area of active research [6-15]. For the fully automated creation of models, tools such as PathwayTools [6], ModelSEED [7], SuBliMinaL [8], FAME [10], RAVEN [12], CoReCo [13], Merlin [14], PyFBA [15], and CarveME [16] are intended to generate a fully functioning first draft reconstruction (FDR), or even first draft model, based on core skeleton template models and/or whole-genome sequence annotations of the organism in question. In-depth comparisons of the capabilities of these tools is available elsewhere [17] and within the original papers presenting the tools.

Some of the tools, such as ModelSeed [7], MicrobesFlux [11], and FAME [10] are web-based tools, whereas others might necessitate a download of databases that they rely on or a list of required additional software.

Of the tools mentioned above, CarveMe [16] has a notably different and novel approach: From a manually curated universal template model, a reconstruction for a particular organism is "carved" using a reaction reduction process based on the genome annotation for the chosen organism [16]. At all times during this reaction-reduction process from the template, the shrinking metabolic network is under the constraint of having to support a specified biomass function. This rapid reconstruction process makes it well suited in situations where a large number of FDRs must be generated rapidly, such as for the modeling of microbial communities, and indeed the tool automates the process of merging single-organism metabolic networks into community-level networks [16].

MEMOSys [9] is a stand alone tool, mainly created for managing, developing and storing GEMs but not primarily for generating them.

The aforementioned reconstruction tools have various strengths, thus offering the modeling community a strong base to create and curate single organism FDRs suitable to a user's needs. In addition to these, more specialized tools exist, such as KEGG2SBML [18], which translates KEGG Metabolic Pathway files into SBML.

However, as is well known within the field, even when using one of the tools capable of automatically generating FDRs, it is a necessity to use a host of other tools or software packages in a manual-curation process to optimize a reconstruction's prediction accuracy [7]: It has been observed that, while automatically generated first draft GEMs may be capable of producing biomass, this ability may not be reflected in a GEM's accuracy in predicting experimental outcomes [19]. Thus, much manual curation work is required in order to refine and optimize a model. Such manual curation steps may include gap-filling (the addition of reactions inferred from the flow of metabolites through the metabolic network), the correction of reactions that are improperly mass or charge balanced (which could result in the spontaneous generation of mass or energy within the system), or the modification of reactions that are thermodynamically infeasible.

In general, it is also necessary to manually add and adjust transport reactions, their presence automatically surmised from experimental evidence of uptake rates, cytosolic activity, or template reconstructions. Finally, the biomass composition function is notoriously difficult to assess correctly, as the biomass composition might be subtly different for two related strains in the same medium, but drastically different for the same strain in two different media [20]. While the maximization of biomass production is a much used primary cellular objective in genome-scale metabolic modeling, this is not necessarily the case in vivo, and therefore deciding on a reasonable cellular objective for a given organism may be far more convoluted than simply finding its biomass composition [20].

There is an increasing interest in the modeling of microbial communities, both due to the fact that it is becoming more and more tractable from a technical standpoint, and 
because of the mounting evidence for the importance of microbiomes in ecology [21], industry [22, 23], and health $[24,25]$. In light of this, it seems that for any application outside of highly controlled environments where a monoculture may be maintained, such as in the lab or in industrial bioreactors, capturing the dynamics of interactions between different populations of microbes is essential to understand the behavior of almost any biological system.

However, constructing GEMs for a microbial community is an arduous and time-consuming process: It is not simply a case of twice the organisms resulting in twice the work. As the number of organisms increases, the complexity of the potential interactions also increases exponentially. In order to save time and effort to make the task more tractable, it is therefore imperative to supply researchers with a set of tools that allow the efficient automation of, at least, parts of this reconstruction process. This is a topic of research and development receiving much attention at current conferences.

As a contribution to fill this capability gap, we created a pipeline within the COBRA Toolbox in Matlab named AutoKEGGRec that only uses the Kyoto Encyclopedia of Genes and Genomes (KEGG) database [26-28] as external input. AutoKEGGRec assembles a draft reconstruction for any given query list of KEGG organisms. Basing itself solely on annotations present in this extensive and actively maintained database rather than on template models, potential historic biases from previous reconstructions (positive or negative) are eliminated. Only reactions known and documented in KEGG to be present in the organism are used within the first draft reconstruction. Adding this function to the COBRA toolbox, the most widely used software suite for constraintbased modeling of GEMs, has as a consequence that no further software installation is required for additional metabolic network curation, and that the scientific community may alter or update the AutoKEGGRec functionality as they see fit.

AutoKEGGRec is easy to use, and there is no need for conversion of model files to work with supported SBML versions and requirements of the COBRA toolbox. Specifically, AutoKEGGRec allows the construction of a large number of draft reconstruction networks, as well as the direct assembly of community reconstructions. In the process of assembling these reconstructions, the AutoKEGGRec function also constructs a consolidated draft metabolic network consisting of a union of all metabolic reactions in the query organisms. Note that analyses may be performed on the consolidated network and other data structures generated by the function.

\section{Implementation}

AutoKEGGRec is designed in Matlab 2017b, hereafter referred to as Matlab, and uses functions within the
COBRA Toolbox v3.0, hereafter referred to as COBRA toolbox/COBRA. Since AutoKEGGRec uses existing COBRA toolbox functions for saving the models, the generated data structures containing the reconstructed model work seamlessly with all COBRA functions. In the following, we will illustrate functions of AutoKEGGRec by using a set of query organisms consisting of the five E. coli K12 strains with the KEGG IDs $e c o, e c j, e c d, e b w$, and $e c o k$ and explain the overall design of the pipeline. A user Manual [Additional file 1] and a matlab version of the tool [Additional file 2] are provided together with the article. AutoKEGGRec will be submitted to be integrated with the COBRA Toolbox.

\section{Overall design}

Designed to be a fast tool that is easy to use, AutoKEGGRec facilitates the rapid assembly of draft models from KEGG annotations for one or several query organisms. Internally, it's designed for efficiency of operation, first minimizing the number of queries sent to the KEGG servers during data collection stage and, subject to this, minimizing the number of operations needed to assemble the draft model from the collected data. Intended to fit into the COBRA toolbox as a function, AutoKEGGRec requires no further installation or setup. While not necessary for its intended operation, it is directly amenable to be modified by each user as required since it is provided as a Matlab function. Figure 1 gives a graphical overview of the different steps involved in the creation of the first draft reconstruction (right green box), and it highlights the additional functionality available using the optional flags (yellow boxes).

AutoKEGGRec needs the input of KEGG organism IDs for the user-chosen organisms as a list of strings followed by the optional flags discussed further in the Manual (leftmost green box in Fig. 1). The pipeline initiates by using the KEGG organism IDs for querying the linkage between enzyme commission (EC) numbers and the corresponding genes from KEGG for each organism, as per step (1) in Fig. 1. In step (2), AutoKEGGRec retrieves the linkage between reactions and EC numbers. Based on the assembled data, the function generates matrices that use EC numbers to store the linkage between KEGG reaction ID and the genes of the organisms. Next, every reaction in KEGG associated with one or more query organisms is retrieved and related to the target organism(s) via corresponding genes (step (3)). This Organisms-ReactionsGenes (ORG) matrix is returned by AutoKEGGRec when submitting the OrgRxnGen-flag (yellow box in Fig. 1), and is thus directly available for further analysis by the user. At this stage, each reaction together with its complete annotation is downloaded from KEGG, as well as the corresponding compound annotations. Since the single organism models and the community model can both 


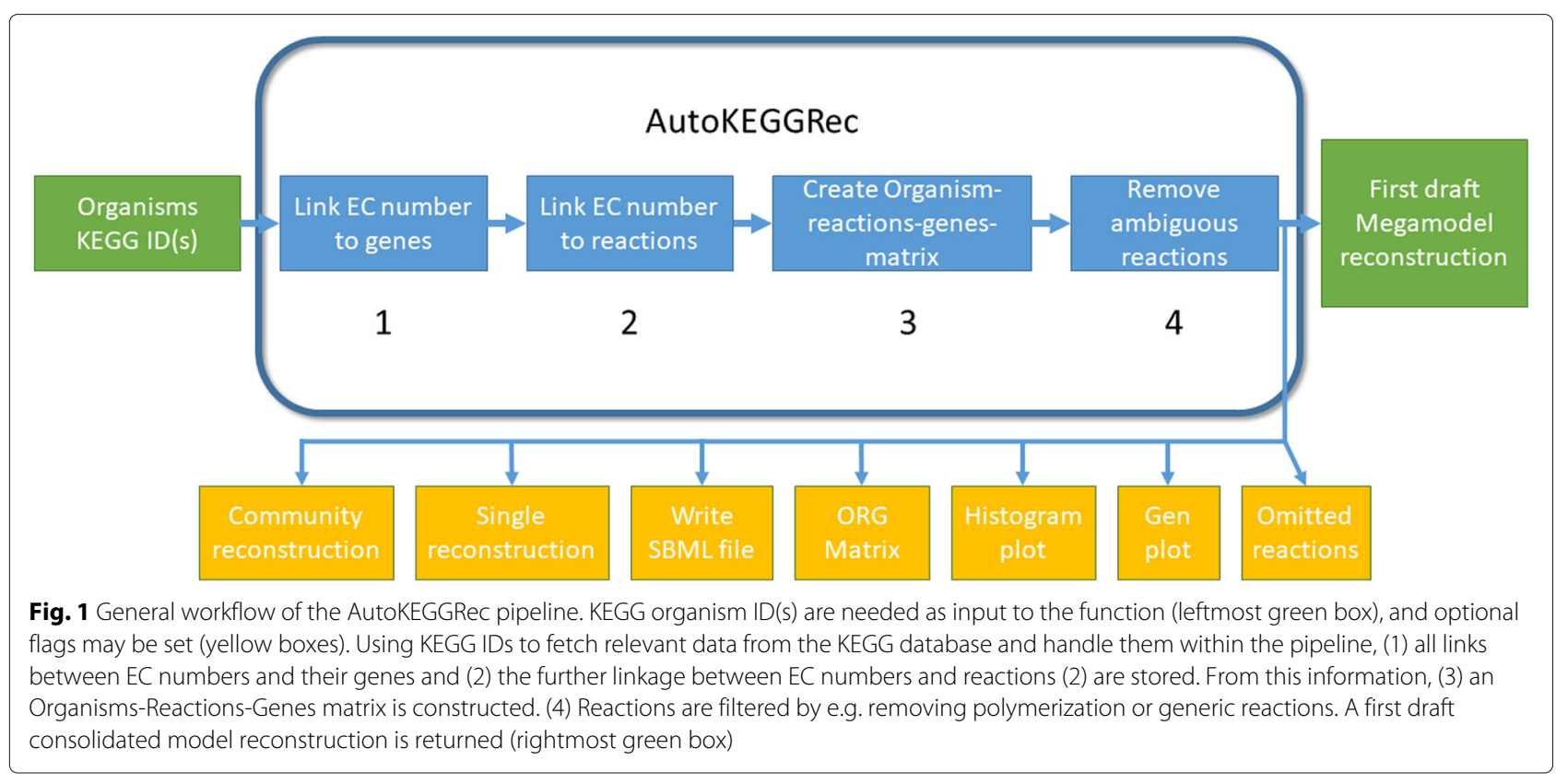

be generated more rapidly using the consolidated model, the latter model is generated by default.

Note that the steps involved in downloading all relevant information from KEGG is generally the most time consuming part of the pipeline, except for the case of large community models consisting of roughly 15 or more organisms, refer to Table 2. Consequently, we have implemented the download process using the Matlab function parfor for parallelization; in anticipation of this, the parpool functionality is initiated by default at the beginning of the function execution and shut down in the end.

All the reactions in the draft metabolic network(s) are filtered to avoid redundant or poorly defined reactions (step (4)). For example, if the "Comment"-field in KEGG contains "Remark" and the reaction equation contains $G$ (glycan instead of a compound name) or $n$ (polymer, generic reaction), the reactions are not included in the reconstruction. If the "Remark" field contains the strings "Generic", "General", or "General reaction", these reactions are also omitted. Additionally, the compound information stored within KEGG occasionally note whether a compound is generic, and lists exact mass. When mass is set to 0 for a compound, this signifies it as a generic compound, and reactions that contain it are omitted from the reconstruction.

Omitted reactions are, for the sake of transparency and potential utility, stored within an additional Matlab structure, which is accessible for the user using the OmittedData option flag. These reactions are marked with a new rxnAttention field which explains why AutoKEGGRec omitted them from the reconstruction. This allows the user to first identify omitted reactions relevant to their project, followed by a curation step before they are potentially included.

In generating an FDR, AutoKEGGRec will add the complete reaction annotations: where possible, annotation fields supported by COBRA are used, but some new fields are, by necessity, created to store the information. Using the createModel function in the COBRA toolbox, the consolidated-type draft reaction network is created based on KEGG genome annotation and linked reactions. The GPR (gene-protein-reaction) rules are added based on the ORG matrix, and the model is annotated according to the KEGG information for the reactions and components. This includes compound names, reaction names, and compound KEGG IDs, to name just a few.

AutoKEGGRec will generate individual draft network reconstructions for each of the listed organisms when the SingleModels option flag is provided. These draft models are generated by first making multiple copies of the consolidated model (one for each organism), before removing reactions not specifically present in each organism. The community model is generated using the COBRA createModel function with the complete list of reactions as input. Here, each organism appears as a separate compartment since AutoKEGGRec will add the organism's KEGG ID to all of its compounds.

Since the resulting output is a Matlab structure variable, the user only needs to define a single output variable independent of the number or combination of optional input flags.

\section{AutoKEGGRec optional settings}

In Fig. 1, the separate option flags are indicated by the presence of yellow boxes, one for each optional setting. 
A discussion and description of the specific functionality provided by the different options is to be found within the Manual. Note that, AutoKEGGRec will create a consolidated reconstruction if no option flags are passed along with the function call as this is the default option. The option flags OrgRxnGen, GenePlot and Histogram may be used without generating a reconstruction, only providing the information listed for these flags (corresponding to steps 1-3 in Fig. 1). In this case, annotations for individual reactions and compounds are not downloaded, allowing AutoKEGGRec to execute significantly faster. Using the option flag OmittedData requires the download of all target reactions from KEGG, but no reconstruction is generated or stored, unless a flag to build one is passed.

\section{Main matrices - Organisms-Reactions-Genes matrix}

The internal workings of AutoKEGGRec rely on a series of arrays and matrices, leveraging Matlab's efficient handling of these data structures. All reactions in KEGG are compiled into an ordered list. To facilitate the fast conversion from KEGG ID back to internal reaction indexing, a reference table is constructed. All genes associated with the organisms in the user-query are fetched from KEGG along with their associations with EC numbers, the full set of linkages between EC numbers, and the reactions they catalyze. In the same way as for the reactions, reference lookups from string IDs to internal list indexes are built, allowing swift translation between these two identifiers.

From these index listings, several binary matrices are constructed. One matrix matches EC numbers to the reactions. Based on this matrix, AutoKEGGRec constructs the Organisms-Reactions (OR) matrix, that matches organisms to reactions. Thus, the OR matrix describes a bipartite graph representing the reactome of each query organism as given by KEGG from the OR matrix, a complete list of every reaction present in at least one of the query organisms is assembled, allowing further KEGG queries to be conducted only for these reactions. Also, AutoKEGGRec constructs the OrganismsReactions-Genes (ORG) matrix using data in the OR matrix

Table 1 shows a subsection of the ORG matrix for $E$. coli $\mathrm{K}-12$ to illustrate the organization of information. Here, each row corresponds to a KEGG reaction ID, and

Table 1 Example output using the OrgRxnGen flag within AutoKEGGRec

\begin{tabular}{|c|c|c|c|c|c|c|c|c|}
\hline KEGG ID & eco & ecj & ecd & ebw & ecok & Sum & Total & Genes \\
\hline R00001 & & & & & & 0 & 0 & 0 \\
\hline R00002 & & & & & & 0 & 0 & 0 \\
\hline R00004 & b4226 & JW4185 & ECDH10B_4421 & BWG_3936 & ECMDS42_3668 & 5 & 1 & 1 \\
\hline R00005 & & & & & & 0 & 0 & 0 \\
\hline R00006 & $\begin{array}{l}\text { b0078, } \\
\text { b3670, } \\
\text { b0077, } \\
\text { b3671, } \\
\text { b3769 }\end{array}$ & $\begin{array}{l}\text { JW0077, } \\
\text { JW3645, } \\
\text { JW0076, } \\
\text { JW3646, } \\
\text { JW3742 }\end{array}$ & $\begin{array}{l}\text { ECDH10B_3853, } \\
\text { ECDH10B_3958, } \\
\text { ECDH10B_3854 }\end{array}$ & $\begin{array}{l}\text { BWG_0073, } \\
\text { BWG_0074, } \\
\text { BWG_3454, } \\
\text { BWG_3361, } \\
\text { BWG_3362 }\end{array}$ & $\begin{array}{l}\text { ECMDS42_3207, } \\
\text { ECMDS42_0071, } \\
\text { ECMDS42_0072, } \\
\text { ECMDS42_3105, } \\
\text { ECMDS42_3106 }\end{array}$ & 5 & 1 & $3 ; 5$ \\
\hline R00008 & & & & & & 0 & 0 & 0 \\
\hline R00009 & $\begin{array}{l}\text { b1732, } \\
\text { b3942 }\end{array}$ & $\begin{array}{l}\text { JW1721, } \\
\text { JW3914 }\end{array}$ & $\begin{array}{l}\text { ECDH10B_1870, } \\
\text { ECDH10B_4131 }\end{array}$ & $\begin{array}{l}\text { BWG_1545, } \\
\text { BWG_3611 }\end{array}$ & $\begin{array}{l}\text { ECMDS42_1407, } \\
\text { ECMDS42_3380 }\end{array}$ & 5 & 1 & 2 \\
\hline R00010 & $\begin{array}{l}\text { b1197, } \\
\text { b3519 }\end{array}$ & $\begin{array}{l}\text { JW3487, } \\
\text { JW1186 }\end{array}$ & $\begin{array}{l}\text { ECDH10B_3696, } \\
\text { ECDH10B_1250 }\end{array}$ & $\begin{array}{l}\text { BWG_3208, } \\
\text { BWG_1022 }\end{array}$ & $\begin{array}{l}\text { ECMDS42_2954, } \\
\text { ECMDS42_0984 }\end{array}$ & 5 & 1 & 2 \\
\hline R00011 & & & & & & 0 & 0 & 0 \\
\hline R00012 & & & & & & 0 & 0 & 0 \\
\hline R00013 & b0507 & JW0495 & ECDH10B_0463 & BWG_0384 & ECMDS42_0400 & 5 & 1 & 1 \\
\hline R00014 & $\begin{array}{l}\text { b0114, } \\
\text { b0078, } \\
\text { b3670, } \\
\text { b0077, } \\
\text { b3671, } \\
\text { b3769 }\end{array}$ & $\begin{array}{l}\text { JW0110, } \\
\text { JW0077, } \\
\text { JW3645, } \\
\text { JW0076, } \\
\text { JW3646, } \\
\text { JW3742 }\end{array}$ & $\begin{array}{l}\text { ECDH10B_0094, } \\
\text { ECDH10B_3853, } \\
\text { ECDH10B_3958, } \\
\text { ECDH10B_3854 }\end{array}$ & $\begin{array}{l}\text { BWG_0073, } \\
\text { BWG_0074, } \\
\text { BWG_3454, } \\
\text { BWG_3361, } \\
\text { BWG_3362, } \\
\text { BWG_0107 }\end{array}$ & $\begin{array}{l}\text { ECMDS42_3207, } \\
\text { ECMDS42_0071, } \\
\text { ECMDS42_0072, } \\
\text { ECMDS42_0105, } \\
\text { ECMDS42_3105, } \\
\text { ECMDS42_3106 }\end{array}$ & 5 & 1 & $4 ; 6$ \\
\hline R00015 & & & & & & 0 & 0 & 0 \\
\hline R00017 & b3518 & JW3486 & ECDH10B_3695 & BWG_3207 & ECMDS42_2953 & 5 & 1 & 1 \\
\hline
\end{tabular}

Here the Organism-Reaction-Gene matrix for the five E. coli K-12 strains is shown. The genes encoding the the first 14 reactions in KEGG, followed by the genes, if any, for the different organisms. Also provided with this output, in the three rightmost columns, is the Sum of organisms whose metabolism contains this reaction, the Total fraction of strains whose metabolism contains this reaction, and the number of Genes for the different organisms for that reaction 
empty rows indicate that the particular reaction ID is not present in any of the organisms (contains a zero entry in the columns Sum, Total, and Genes). In this example, a value of five in the Sum column and a value of unity in the Total column shows that the reaction is present in all E. coli K-12 strains. The genes associated with each reaction in the organisms are separated with, an "OR"-relationship. The number of genes per reaction is summarized in the "Genes" column; if the value varies between the organisms, a list of the different values are reported.

\section{Critical issues}

Note that AutoKEGGRec is not intended to produce a fully functioning model, but a first draft reconstruction based on the KEGG database through a set of simple and transparent procedures. There are a few things to keep in mind:

- The pipeline uses only KEGG organism IDs as input.

- Glycan reactions are mostly removed. Since some of the glycans also have compound numbers within KEGG, these are still included in the first draft reconstruction. Reactions containing only glycans, i.e. KEGG metabolite IDs starting with $G$ instead of $C$, are not included in the reconstruction even if there is no alternative reaction.

- Polymer reactions or non-specific reactions dealing with an of amount $n$ or $m$ of a compound $C$ are not included by AutoKEGGRec: The constraint-based modeling is based on a steady-state or semi-steady-state approximation, and there is no consensus in the field for how one handles polymerization reactions. The user may edit these reactions to allow their inclusion in a reconstruction.

- There is no assignment of reactions to different compartments. All reactions and compounds are placed in the default compartment "cytosol" by the COBRA function createModel. Even though KEGG includes a few transport reactions for some organisms, these are not explicitly marked.

- Genes are linked to reactions. In the case of more than one gene associated with a reaction, the genes are linked together with "OR"-relationships since KEGG does not specify how the gene-products interact. Consequently, the user must manually curate these relations, providing the correct "AND" or "OR" relations between genes.

- Reactions with massless compounds are omitted. This step is necessary to maintain a mass balance within the reconstruction. Some of these reactions contain compounds like "protein" (C00017) or "DNA" (C00039), but also more specific compounds like "DNA adenine" (C00821).

\section{Results}

Our implementation of an FDR pipeline fully integrated with the much used COBRA toolbox for Matlab makes it an easily accessible resource for the constraint-based modelling community. By basing the reconstruction of the FDRs solely on the KEGG database, the results reflect the quantity and quality of the KEGG curation, which is actively updated and maintained by the community. Note that, because of the lack of directionality of annotations in KEGG, all reactions are reversible in the FDR. In most cases, this choice will not coincide with biological reality; however, there exists COBRA functions to estimate the reaction directionality according to thermodynamics $[29,30]$.

Reactions are mass and charge balanced according to the KEGG reaction annotations, which are generally of high quality. We note that the manual determination of e.g. biomass reaction, transport reactions, and reaction directionality, are all expected steps in the process of transforming an automatically generated FDR to a highquality curation. With AutoKEGGRec, the source and assembly of the metabolic network data is clear and all contained in the KEGG namespace, forming a solid foundation upon which to refine and curate the model, making it a well-suited alternative for proficient COBRA toolbox users.

In Table 2, the timings and sizes of the giant components for a range of test examples are listed. We note that the percentage of all reactions found as part of the giant component for the various draft reconstructions is close to 99\%; the resulting FDRs are graphically represented in Fig. $2 \mathrm{a}$ and b. This speaks to the quality of the KEGG database and its annotations and to the promise of FDR assembly from high-quality curated databases.

The runtime of AutoKEGGRec is, for most applications, dominated by the reaction and compound retrieval-time from KEGG (seen in Table 2).

The number of omitted reactions for the five mentioned E. coli K-12 strains is 519 . Of these, 141, about $8.08 \%$ of the 1745 reactions associated with all the $E$. coli $\mathrm{K}-12$ strain genes in KEGG, were removed based on reaction filtering. Of these, 48 were generic reactions, 6 are polymerization reactions, and 53 include glycans and are therefore not stored in the FDR. In the latter case, alternative reactions containing the KEGG IDs compound names are stored within the FDR if they exist in KEGG. A total of 31 reactions were removed from the FDR for being ill-defined (containing the same compound on both sites of the reaction equation), and 3 reactions were excluded from the FDR because of potentially being polymerization reactions. AutoKEGGRec evaluates the 1626 compounds of the remaining 1604 reactions, to identify generic (mass-less) compounds. In the example case of the E. coli $\mathrm{K}-12$ 
Table 2 Average execution time for AutoKEGGRec within Matlab 2017b on a Dell Latitude 7490 with an 17-7600U using 2-core-parallelization

\begin{tabular}{|c|c|c|c|}
\hline Tested strains incl. number of organisms & Mean runtime $[s]$ & Reactions/metabolites & Giant component $[\#, \%]$ \\
\hline E. coli K-12, 5 KEGG IDs & $2,995 \pm 65$ & $1226 / 1188$ & $1215 / 99.10 \%$ \\
\hline P. putida, 12 KEGG IDs & $3,957 \pm 166$ & $1412 / 1445$ & $1400 / 99.15 \%$ \\
\hline B. subtilis, 15 KEGG IDs & $3,538 \pm 216$ & $1108 / 1134$ & $1103 / 99.55 \%$ \\
\hline E. coli, 20 KEGG IDs & $4,792 \pm 289$ & $1342 / 1302$ & $1331 / 99.18 \%$ \\
\hline E. coli, 25 KEGG IDs & $5,618 \pm 322$ & $1351 / 1312$ & $1340 / 99.19 \%$ \\
\hline E. coli, 30 KEGG IDs & $6,724 \pm 130$ & $1354 / 1316$ & $1343 / 99.19 \%$ \\
\hline E. coli, 35 KEGG IDs & $7,750 \pm 208$ & $1358 / 1319$ & $1347 / 99.19 \%$ \\
\hline E. coli, 40 KEGG IDs & $9,115 \pm 198$ & $1359 / 1321$ & $1348 / 99.19 \%$ \\
\hline E. coli, 45 KEGG IDs & $10,032 \pm 651$ & $1359 / 1321$ & 1348/99.19\% \\
\hline E. coli, 50 KEGG IDs & $11,466 \pm 1,065$ & $1359 / 1321$ & $1348 / 99.19 \%$ \\
\hline E. coli, 55 KEGG IDs & $12,687 \pm 1,269$ & $1359 / 1321$ & $1348 / 99.19 \%$ \\
\hline E. coli, 60 KEGG IDs & $13,848 \pm 1,658$ & $1359 / 1321$ & 1348/99.19\% \\
\hline E. coli, 65 KEGG IDs & $15,082 \pm 1,850$ & $1360 / 1323$ & 1349/99.19\% \\
\hline
\end{tabular}

The columns are: a specification of the queried organisms, the mean and standard deviation runtime (five separate software executions) per dataset using ConsolidatedRec, SingleRecs, CommunityRec, OrgRxnGen, OmittedData and DisconnectedReactions as optional flags, the number of reactions and metabolites in the consolidated FDR and the number and fraction of reactions in the consolidated network's giant component

strains this is true for 390 compounds, which is about $23.98 \%$. These compounds participate in 378 reactions, which increases the number of omitted reactions to 519 (29.74\%), and leaves the FDR with 1226 high quality reactions. All the mentioned reactions are detailed in the output data structure generated by the omittedData option flag, the omitted compounds and the saved KEGG annotations are stored as well, examples of outputs of the omitted data are shown in the Manual.

As an example use of our proposed model field "RxnAttention", 10 reactions were marked in the generated FDR. One such reaction is

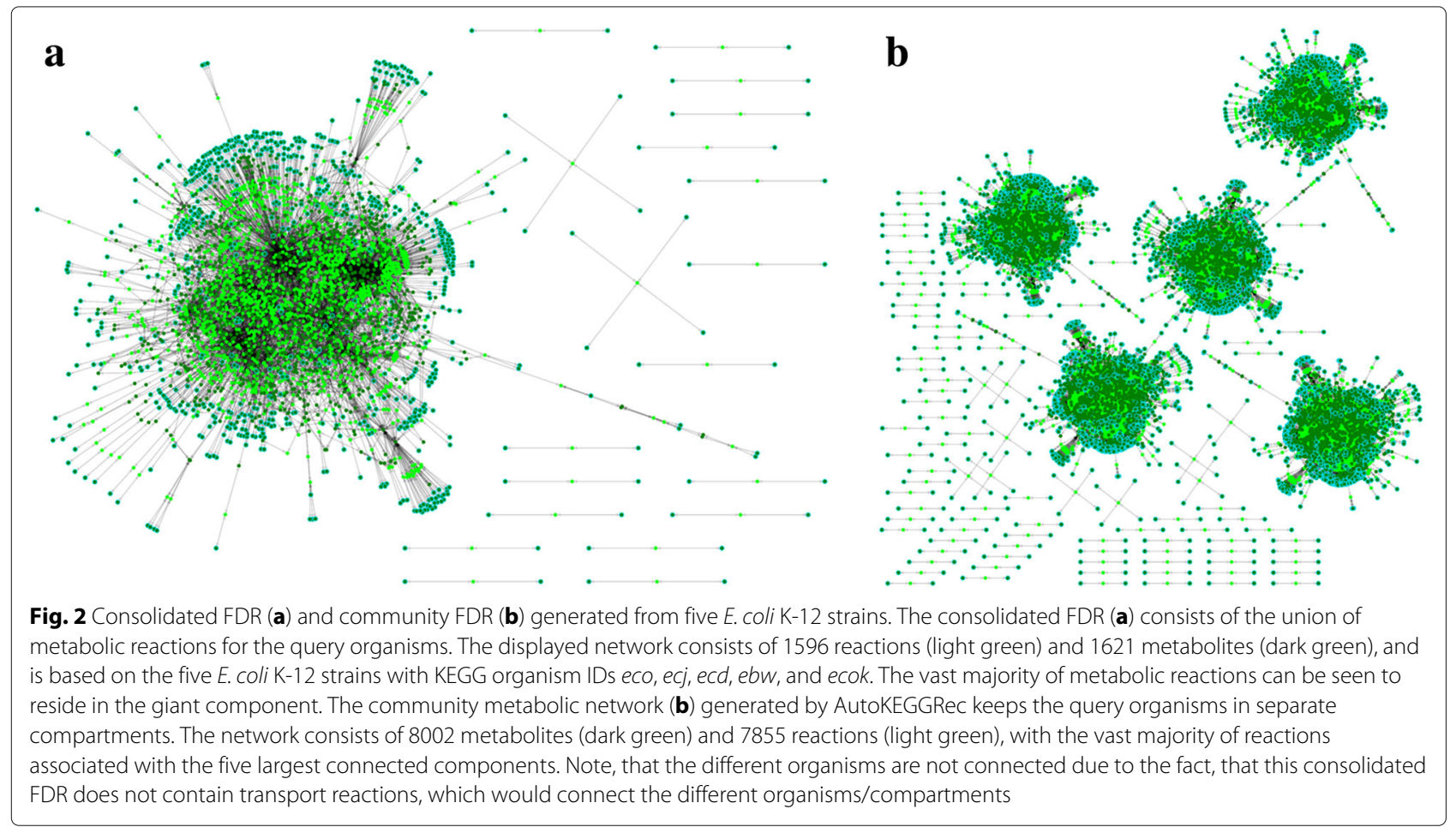




$$
R 05994: G 00124+C 00001 \Leftrightarrow G 00123+C 00124,
$$

which has two glycan compounds. In this case, these compounds correspond to the "normal" KEGG compound IDs C06136 and C06135, leading to an alternative reaction in the KEGG reaction universe using only these $C$ compound names as

$$
R 05112: C 06136+C 00001 \Leftrightarrow C 06135+C 00124 .
$$

Here, the reaction containing only the $C$-named KEGG compounds is used in the FDR generated by AutoKEGGrec. This particular reaction, however, contains the compound C06136, which is, according to the KEGG compound annotation, a generic compound with the formula $\mathrm{C}_{45} \mathrm{H}_{79} \mathrm{~N}_{2} \mathrm{O}_{23} \mathrm{R}$, and for that reason has no mass. While assembling this important compound information, such compounds are identified and the reaction will be marked in the "RxnAttention" field with "This reactions contains a generic compound or a compound without mass in KEGG", so that these omitted reactions are easy do identify. These compounds are stored within the Matlab structure for omitted data so that the user can quickly access the compounds in questions, identify the reason for omission, and decide if and how the user wants to implement the compound/reaction into the (curated) reconstruction. Note that the field "RxnAttention" will be found within the model for included reactions for careful inspection, shown in the next example, as well as within the omitted reactions.

There are examples of glycan molecules which do not have a $C$ compound KEGG ID, e.g. in the reaction

$$
\text { R07807 : G01977 + C00001 } \Leftrightarrow \text { G13073 + C00124. }
$$

It is added to the FDR because of a lack of alternative reactions, and will be marked in the "rxnAttention" annotation field. We encourage users to carefully inspect reactions with this tag that stay within the reconstruction. Additionally, we note that some glycan compounds do not have and exact_mass or mol_mass listed in KEGG, but only mass. However since there is a specific mass given which allows the user to manually check the reactions for mass consistency, AutoKEGGRec includes these compounds.

AutoKEGGRec has no intrinsic limit on the number of query organisms, all 65 E. coli strains stored in KEGG by their KEGG organism ID could be joined into one consolidated model, offering the user the union of the E. coli reaction networks available in KEGG along with corresponding gene links. Even unrelated organisms could be added to include their reactions, pathways, and related gene names.

Directly investigating the correspondence between the AutoKEGGRec FDRs and experimental data would make little sense, as the tool's purpose is not to make a complete model capable of producing biomass. However, in order to give an idea of the amount of remaining work for any modeler employing the tool, we chose to compare the AutoKEGGRec FDR, created using the KEGG ID eco, to the published E. coli models iAF1260 [31] and iJO1366 [32], as well as a model made using ModelSeeds with default settings based on the stored data on E. coli $\mathrm{K}-12$ MG1566 with the ID 511145.180. Comparing the quality of different GEMs is a complicated issue, not least in part due to differences in namespace, making alignment of different metabolic networks difficult.

Since two of the models are manually curated and the third is made from a template, they contain biomass and transport reactions. As AutoKEGGRec reconstructions do not, such reactions have been removed before comparison. That way the pure reaction networks can be compared to that of the KEGG-based reconstruction generated by AutoKEGGRec. The results can be seen in Table 3. Here, the models are listed with a direct comparison of the different chosen graph parameters, as well as other comparable parameters. Two separate numbers of reactions are given. They are marked with "BR" and "AR", meaning before and after removal of transport reactions, respectively. The rest of the numbers are retrieved after removing such reactions. Some parameters are given for the metabolic network; these are the number of reactions in the reconstruction, the mean degree, and the mean shortest path for each model. Furthermore, the number of blocked metabolites is shown, as well as the number of metabolites occurring in exactly two reactions with the same sign in the S-matrix, is shown. As the transport reactions have been removed, a blocked metabolite is here defined as a metabolite occurring in exactly one

Table 3 Comparison of eco, the FDR generated by AutoKEGGRec, with iAF1260 [31], iJO1366 [32], and ModelSeed model 511145.180 generated with default settings

\begin{tabular}{lllll}
\hline Comparison & eco & iAF1260 & iJO1366 & 511145.180 \\
\hline \# of reactions BR & 1224 & 2382 & 2583 & 1635 \\
\# of metabolites BR & 1185 & 1668 & 1805 & 1573 \\
\# of reactions AR & 1224 & 2081 & 2256 & 1510 \\
\# of metabolites AR & 1185 & 1668 & 1805 & 1573 \\
network mean degree & 4.203 & 4.727 & 4.765 & 4.527 \\
network mean shortest path & 4.470 & 4.549 & 4.493 & 3.860 \\
\# of blocked metabolites & 519 & 369 & 390 & 746 \\
\# of same-sign-metabolites & 160 & 89 & 84 & 136 \\
\# of genes in model & 1263 & 1260 & 1366 & 1139 \\
\# of shared genes with eco & 1263 & 927 & 981 & 794
\end{tabular}

We removed all transport, biomass, and ATP maintenance reactions from iAF1260, iJO1366 and 511145.180. Except for reported values marked "BR" (before removal) and "AR" (after removal), results are for the reduced models. Definition of "blocked metabolites" and "same-sign-metabolites" is provided in the main text 
reaction. Combined with the number of metabolites occurring in exactly two reactions, having the same sign in the S-matrix on both of them, they summarize the amount of potentially blocked metabolites. Additionally, the number of genes within each reconstruction, and, if possible due to namespace, the overlap with the AutoKEGGRec reconstruction in terms of number of genes are given. Summarizing the different properties of the models, it seems that $e c o$, the FDR generated in AutoKEGGRec, is of comparable quality to the manually curated models iAF1260 and iJO1366. This speaks to the quality of KEGG, as well as FDRs generated using AutoKEGGRec.

\section{Discussion}

AutoKEGGRec is an easy-to-use tool for the rapid assembly of first-draft reconstructions for genome-scale metabolic modeling including as much data as possible. While there exists a selection of tools and toolkits that aid in the assembly and refinement of first-draft GEMs, these tend to be stand-alone and/or online, both of which, especially in combination with small development teams in temporary academic positions, may cause any number of issues due to lack of support. These tools mostly create single-organism FDRs, as most are not designed for or allow the creation of community or generic/consolidated models.

Any automatic reconstruction necessitates steps such as gap-filling, mass and charge balance for reactions, reaction reversibility corrections, and adjustment of exchange, ATP maintenance and biomass reactions. These steps being mostly automated might appear as a convenience, but it is important to note that these tools often make implicit assumptions in the course of the reconstruction work without marking them as such. This is done in order to achieve functional models, i.e. models that are capable of producing the constituents of a biomass reaction, but, importantly, there are usually many ways of achieving this, and which way is chosen matters for the correctness and quality of the model, making control and traceability of the process an important issue. Many such educated guesses are based on template models or common knowledge, such as the inclusion of seemingly universally essential cofactors [33] in the biomass function. However, the way in which this information is retrieved and assembled is often not completely transparent, leaving important details of the model's workings outside of a user's control or awareness. Any resulting FDR may therefore contain misleading information, which is further propagated in reconstruction projects.

Biomass reactions in particular are known to be fickle with regards to cofactor coefficients [34]. This may lead to false growth predictions with regards to media and knockouts when naively propagated from skeleton models. Therefore, the manual curation of all reactions, and especially the biomass reaction, is still the recommended approach for most applications, and the added value of including template features such as biomass functions is debatable.

To varying degrees, these published tools automate the generation of first draft reconstructions, and greatly facilitate the reconstruction of GEMs. AutoKEGGRec, however, is not intended to automatically generate a first draft model with all requisite transport reactions and a biomass function. Since all information is based only on KEGG, every detail is eminently traceable, which may be of particular interest to modelers, as many "new" models are based on previous models, and may therefore inherit previous poor annotations and assumptions. The intent is to generate an unbiased and clean FDR of the metabolic network based only on the genome annotation in KEGG.

It is implemented as a Matlab function fully integrated with the COBRA toolbox, with its open, communitymaintained and state-of-the-art suite of tools for model curation and manipulation. Additionally, AutoKEGGRec contains the complete annotation for all reactions and compounds offered by KEGG, putting more information right at the modeler's fingertips than what is offered by most other comparable reconstruction tools.

In its rapid assembly of a draft reconstruction, AutoKEGGRec mostly covers the currently automatable parts of the first stage in the protocol to generate high-quality genome-scale metabolic reconstructions [35]. Additionally, it allows not only the creation of an FDR for a single organism, but also consolidated and community FDRs. These features set AutoKEGGRec apart from published methods. Consequently, it is a valuable tool for the current-day practitioner of genome-scale metabolic modeling and reconstruction.

While the reconstruction of microbial consortia remains an immature method, AutoKEGGRec can help speed up the process. Further work in assembling the reconstruction is still expected to rise steadily with the number of organisms, as not only does each separate organism's metabolic network needs to be curated, but also the interactions between the different networks and their global effect on the environment. However, due to the chosen naming convention for compartments within the community models, transport reactions can easily be added simultaneously for multiple organisms if necessary.

\section{Conclusion}

Here we present AutoKEGGRec, a fast tool to be used within the COBRA toolbox in Matlab that is able to create single organism, community, and consolidated first draft reconstructions based on the KEGG database. Unlike most other available tools, AutoKEGGRec is designed from the ground up to allow for community and consolidated metabolic network reconstructions. Also, being 
based only on data from KEGG, it has a clear and transparent data ancestry.

AutoKEGGRec does not provide a fully functioning model since elements such as a biomass function, ATP maintenance function, and most transport reactions for the given organism(s) are not included. The addition of these elements would require estimations and guesswork beyond the data available in KEGG at present. However, AutoKEGGRec does provide a very well annotated first draft reconstruction based on all data available in KEGG. As with reconstructed networks generated with any software package, the user should perform additional experiments, and add biomass function(s), ATP maintenance, and relevant import reactions to further enhance the reconstruction; all tasks which may be performed using existing functions in the COBRA toolbox. Consequently, AutoKEGGRec fills an important niche for the modeling community with its reliable and rapid generation of well annotated single, community, and consolidated FDRs within the COBRA toolbox environment based only on the clear, high-quality, and freely accessible data in KEGG.

\section{Availability and requirements \\ Project name: AutoKEGGRec \\ Operating system(s): Any which can run Matlab \\ Programming language: Matlab}

Other requirements: Matlab, COBRA Toolbox version 3.0 or newer

\section{License: Matlab}

Any restrictions to use by non-academics: Licence needed for Matlab

An internet connection is required.

Availability: AutoKEGGRec will be submitted to be part of the COBRA toolbox. Additionally, the software is included as supplementary file, and is also freely available for download at https://www.ntnu.edu/almaaslab and https://github.com/emikar/AutoKEGGRec. AutoKEGGRec was developed and tested on Matlab (versions 2017a, 2017b and 2018a) and the COBRA toolbox v3.0.

\section{Additional files}

Additional file 1: User manual for AutoKEGGRec. (PDF $5258 \mathrm{~kb}$ )

Additional file 2: The Matlab file for AutoKEGGRec. (M 83 kb)

\section{Abbreviations}

COBRA: COnstraint-Based Reconstruction and Analysis; EC number: Enzyme Commission number; FDR: First Draft Reconstruction; GEMs: Genome-Scale Metabolic modelS; GPR: Gene Protein Relation; KEGG: Kyoto Encyclopedia of Genes and Genomes; OR: Organisms-Reactions; ORG:

Organisms-Reactions-Genes

\section{Acknowledgements}

The authors would like to thank Dr. A. Heinken, Dr. A. Voigt, N. Martyshenko, P. Røynestad, T. Kumelj, S. Sulheim and M. Hall for helpful comments and suggestions.

\section{Funding}

EK is funded by the Norwegian Research Council project \#269084, and CS is funded by the Norwegian Research Council project \#271585. The funders had no role in study design, data collection and analysis, decision to publish, or preparation of the manuscript.

\section{Availability of data and materials}

The software is included as supplementary file, and latest version updates are also freely available for download at https://www.ntnu.edu/almaaslab and https://github.com/emikar/AutoKEGGRec.

\section{Author's contributions}

EK and CS conceived the tool. EK, CS, and EA developed the project. EK and CS programmed and tested the tool. EK, CS, and EA analyzed the data. All authors wrote and approved the final manuscript.

\section{Ethics approval and consent to participate}

Not applicable.

\section{Consent for publication}

Not applicable.

\section{Competing interests}

The authors declare that they have no competing interests.

\section{Publisher's Note}

Springer Nature remains neutral with regard to jurisdictional claims in published maps and institutional affiliations.

Received: 13 April 2018 Accepted: 6 November 2018

Published online: 04 December 2018

References

1. Feist AM, Herrgård MJ, Thiele I, Reed JL, Palsson BØ. Reconstruction of biochemical networks in microorganisms. Nat Rev Microbiol. 2009;7(2): 129.

2. Oberhardt MA, Puchalka J, Martins dos Santos VAP, Papin JA. Reconciliation of genome-scale metabolic reconstructions for comparative systems analysis. PLoS Comput Biol. 2011;7(3):1001116.

3. Pfau T, Pacheco MP, Sauter T. Towards improved genome-scale metabolic network reconstructions: unification, transcript specificity and beyond. Brief Bioinforma. 2016;17(6):1060-9.

4. Heirendt L, Arreckx S, Pfau T, Mendoza SN, Richelle A, Heinken A, Haraldsdottir HS, Keating SM, Vlasov V, Wachowiak J, et al. Creation and analysis of biochemical constraint-based models: the cobra toolbox v3. 0 . arXiv:1710.04038 [q-bio.QM]. 2017

5. Schellenberger J, Que R, Fleming RM, Thiele I, Orth JD, Feist AM, Zielinski DC, Bordbar A, Lewis NE, Rahmanian S, et al. Quantitative prediction of cellular metabolism with constraint-based models: the cobra toolbox v2. 0. Nat Protoc. 2011;6(9):1290

6. Karp PD, Paley S, Romero P. The pathway tools software. Bioinformatics. 2002:18(suppl_1):225-32.

7. Henry CS, DeJongh M, Best AA, Frybarger PM, Linsay B, Stevens RL. High-throughput generation, optimization and analysis of genome-scale metabolic models. Nat Biotechnol. 2010;28(9):977.

8. Swainston N, Smallbone K, Mendes P, Kell DB, Paton NW. The subliminal toolbox: automating steps in the reconstruction of metabolic networks. J Integr Bioinforma. 2011;8(2):187-203.

9. Pabinger S, Rader R, Agren R, Nielsen J, Trajanoski Z. Memosys: Bioinformatics platform for genome-scale metabolic models. BMC Syst Biol. 2011:5(1):20.

10. Boele J, Olivier BG, Teusink B. Fame, the flux analysis and modeling environment. BMC Syst Biol. 2012;6(1):8.

11. Feng X, Xu Y, Chen Y, Tang YJ. Microbesflux: a web platform for drafting metabolic models from the kegg database. BMC Syst Biol. 2012;6(1):94.

12. Agren R, Liu L, Shoaie S, Vongsangnak W, Nookaew I, Nielsen J. The raven toolbox and its use for generating a genome-scale metabolic model for penicillium chrysogenum. PLoS Comput Biol. 2013;9(3):1002980.

13. Pitkänen $E$, Jouhten $P$, Hou J, Syed MF, Blomberg P, Kludas J, Oja M, Holm L, Penttilä M, Rousu J, et al. Comparative genome-scale reconstruction of gapless metabolic networks for present and ancestral species. PLoS Comput Biol. 2014;10(2):1003465. 
14. Dias O, Rocha M, Ferreira EC, Rocha I. Reconstructing genome-scale metabolic models with merlin. Nucleic Acids Res. 2015;43(8):3899-910.

15. Cuevas DA, Edirisinghe J, Henry CS, Overbeek R, O'Connell TG, Edwards RA. From dna to fba: how to build your own genome-scale metabolic model. Front Microbiol. 2016;7:907.

16. Machado D, Andrejev S, Tramontano M, Patil KR. Fast automated reconstruction of genome-scale metabolic models for microbial species and communities. Nucleic Acids Res. 2018;46(15):7542-53.

17. Hamilton JJ, Reed JL. Software platforms to facilitate reconstructing genome-scale metabolic networks. Environ Microbiol. 2014;16(1):49-59.

18. Funahashi A, Jouraku A, Kitano H. Converting KEGG pathway database to SBML. In: 8th Annual International Conference on Research in Computational Molecular Biology (RECOMB); 2004. http://sbml.org/ Software/KEGG2SBML. Accessed 4 Jan 2018.

19. Heinken A, Sahoo S, Fleming RM, Thiele I. Systems-level characterization of a host-microbe metabolic symbiosis in the mammalian gut. Gut Microbes. 2013;4(1):28-40.

20. Feist AM, Palsson BO. The biomass objective function. Curr Opin Microbiol. 2010;13(3):344-9.

21. Larsen P, Hamada Y, Gilbert J. Modeling microbial communities: Current, developing, and future technologies for predicting microbial community interaction. J Biotechnol. 2012;160(1-2):17-24.

22. Green TJ, Smullen R, Barnes AC. Dietary soybean protein concentrate-induced intestinal disorder in marine farmed atlantic salmon, salmo salar is associated with alterations in gut microbiota. Vet Microbiol. 2013;166(1-2):286-92.

23. Parnell JJ, Berka R, Young HA, Sturino JM, Kang Y, Barnhart D, DiLeo MV. From the lab to the farm: an industrial perspective of plant beneficial microorganisms. Front Plant Sci. 2016;7:1110

24. Clemente JC, Ursell LK, Parfrey LW, Knight R. The impact of the gut microbiota on human health: an integrative view. Cell. 2012;148(6): 1258-70.

25. Magnúsdóttir S., Heinken A, Kutt L, Ravcheev DA, Bauer E, Noronha A, Greenhalgh K, Jäger C., Baginska J, Wilmes P, et al. Generation of genome-scale metabolic reconstructions for 773 members of the human gut microbiota. Nat Biotechnol. 2017;35(1):81.

26. Kanehisa M, Goto S. Kegg: kyoto encyclopedia of genes and genomes. Nucleic Acids Res. 2000;28(1):27-30.

27. Kanehisa M, Sato Y, Kawashima M, Furumichi M, Tanabe M. Kegg as a reference resource for gene and protein annotation. Nucleic Acids Res. 2015:44(D1):457-62.

28. Kanehisa M, Furumichi M, Tanabe M, Sato Y, Morishima K. Kegg: new perspectives on genomes, pathways, diseases and drugs. Nucleic Acids Res. 2016;45(D1):353-61.

29. Noor E, Haraldsdóttir HS, Milo R, Fleming RM. Consistent estimation of gibbs energy using component contributions. PLoS Comput Biol. 2013;9(7):1003098.

30. Haraldsdóttir H, Thiele I, Fleming RM. Quantitative assignment of reaction directionality in a multicompartmental human metabolic reconstruction. Biophys J. 2012;102(8):1703-11.

31. Feist AM, Henry CS, Reed JL, Krummenacker M, Joyce AR, Karp PD, Broadbelt $\sqcup$, Hatzimanikatis $V$, Palsson $B \varnothing$. A genome-scale metabolic reconstruction for escherichia coli k-12 mg1655 that accounts for 1260 orfs and thermodynamic information. Mol Syst Biol. 2007;3(1):121.

32. Orth JD, Conrad TM, Na J, Lerman JA, Nam H, Feist AM, Palsson B. Ø. A comprehensive genome-scale reconstruction of escherichia coli metabolism-2011. Mol Syst Biol. 2011;7(1):535.

33. Xavier JC, Patil KR, Rocha I. Integration of biomass formulations of genome-scale metabolic models with experimental data reveals universally essential cofactors in prokaryotes. Metab Eng. 2017;39:200-8.

34. MacGillivray M, Ko A, Gruber E, Sawyer M, Almaas E, Holder A. Robust analysis of fluxes in genome-scale metabolic pathways. Sci Rep. 2017;7(1): 268.

35. Thiele I, Palsson B. Ø. A protocol for generating a high-quality genome-scale metabolic reconstruction. Nat Protoc. 2010;5(1):93.

\section{Ready to submit your research? Choose BMC and benefit from}

- fast, convenient online submission

- thorough peer review by experienced researchers in your field

- rapid publication on acceptance

- support for research data, including large and complex data types

- gold Open Access which fosters wider collaboration and increased citations

- maximum visibility for your research: over $100 \mathrm{M}$ website views per year

At BMC, research is always in progress.

Learn more biomedcentral.com/submissions 